\title{
Nanocrystalline GaN and GaN:H Films Grown by RF-Magnetron Sputtering
}

\author{
D. M. G. Leite*, A. L. J. Pereira, L. F. da Silva, and J. H. Dias da Silva \\ Laboratório de Filmes Semicondutores, Depto de Física, \\ Universidade Estadual Paulista, 17033-360 - Bauru-SP, Brazil
}

Received on 8 December, 2005

\begin{abstract}
The structural and optical properties of nanocrystalline $\mathrm{GaN}$ and $\mathrm{GaN}: \mathrm{H}$ films grown by RF-magnetron sputtering are focused here. The films were grown using a Ga target and a variety of deposition parameters $\left(\mathrm{N}_{2} / \mathrm{H}_{2}\right.$ /Arflow rates, RF power, and substrate temperatures). $\mathrm{Si}(100)$ and fused silica substrates were used at relatively low temperatures $\left(\mathrm{T}_{s} \leq 420 \mathrm{~K}\right)$. The main effects resulting from the deposition parameters variations on the films properties were related to the presence of hydrogen in the plasma. The X-ray diffraction analysis indicates that the grain sizes $(\sim 15 \mathrm{~nm})$ and the crystallized volume fraction significantly decrease when hydrogen is present in the plasma. The optical absorption experiments indicate that the hydrogenated films have absorption edges very similar to that of $\mathrm{GaN}$ single crystal films reported in the literature, while the non-hydrogenated samples present larger absorption tails encroaching into the gap energies.
\end{abstract}

Keywords: GaN; GaN:H; Nanocrystalline; Sputtering; Hydrogenation

\section{INTRODUCTION}

Gallium nitride films received considerable attention in the last years due to their applications in optoelectronic devices $[1,2]$. Several reports are found for different material structures: single crystal [2-4], nanocrystalline [5,6], and amorphous [7]. Most studies refer to film preparation onto different substrates due to the difficulties concerning the growth of GaN bulk crystals [2-4].

Recently, Guo et al. [8] have successfully grown single crystal GaN films at relatively low substrate temperatures using the RF-magnetron sputtering, bringing considerable interest to the preparation using this technique. The sputtering technique is versatile for making possible the deposition of a large variety of films on different types of substrates over large areas and at relatively low temperatures. The simplicity of the sources used, the wide variety of materials addressed, the low cost, and the generation of fewer residues than some traditional methods, are among the advantages of the technique.

The effect of hydrogenation/de-hydrogenation of GaN was analyzed by several authors, but the analysis are almost [6] completely restricted to the passivation of defects and impurities in single crystal GaN films $[9,10]$.

In this study was reported the preparation of nanocrystalline $\mathrm{GaN}$ and $\mathrm{GaN}$ :H thin films grown by RF-magnetron sputtering with different deposition parameters $\left(\mathrm{N}_{2} / \mathrm{H}_{2} / \mathrm{Ar}\right.$ flow rates, RF power and substrate temperatures). The effects of some deposition parameter variations on the film properties were analyzed by X-ray diffraction and optical absorption. The main results were related to hydrogen incorporation.

\section{EXPERIMENTAL DETAILS}

The films were prepared in a RF-magnetron sputtering setup specially assembled to grow III-V compound semiconductor films [11]. The system used electronically controlled gas flow, total pressure, and RF power (at $13.6 \mathrm{MHz}$ ). The knife-edge metal seals and turbomolecular pumping allowed residual pressures were better than $1 \times 10^{-5}$ Pa before depositions. Fig 1 shows the schematic representation of the sputtering chamber.

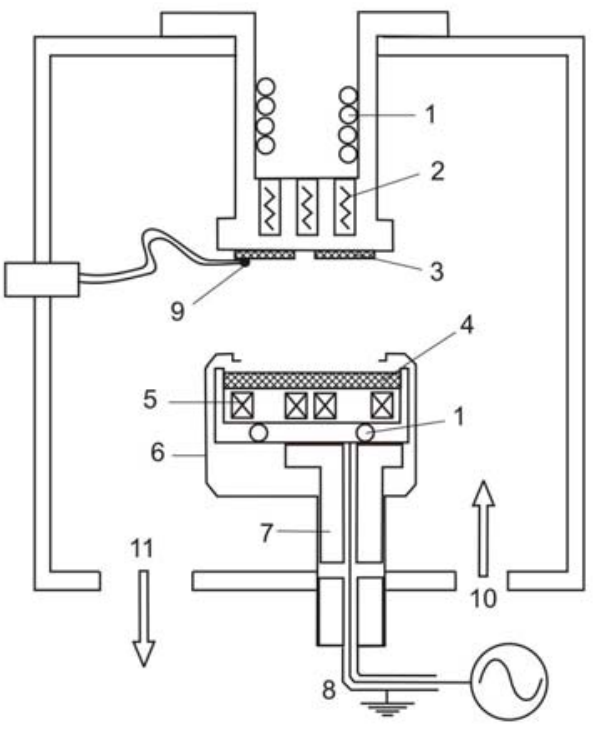

FIG. 1: Schematic representation of the sputtering chamber geometry and main components: 1-Water cooling; 2-Heating resistors; 3Substrates; 4-Ga target; 5-Permanent magnets; 6-Shield; 7-Insulator; 8-RF cable; 9-Thermocouple; 10-Gas inlet; 11-Pumping system.

The $350 \mathrm{~mm}$ diameter chamber shelters a central magnetron target holder of $100 \mathrm{~mm}$ diameter (Fig. 1). The distance between the target surface and the substrate was $50 \mathrm{~mm}$ during all the depositions.

A metallic Ga target ( $7 \mathrm{~N}$-purity) and different $\mathrm{N}_{2} / \mathrm{H}_{2} /$ Ar gas flow ratios (6N-purity) were used in the sputtering process. In each deposition different substrates $(\mathrm{Si}(100)$ and amorphous 
TABLE I: Deposition parameters and the optical gap of $\mathrm{GaN}$ and $\mathrm{GaN}: \mathrm{H}$ films deposited by reactive RF-magnetron sputtering. The total pressure in the chamber was kept constant at 2.0 Pa. The optical gap was set as the energy in which the absorption coefficient is $10^{4} \mathrm{~cm}^{-1}$.

\begin{tabular}{|c|c|c|c|c|c|c|c|c|c|c|}
\hline \multirow{2}{*}{ Name } & \multirow{2}{*}{ Sample } & \multirow{2}{*}{ RF Power } & \multirow{2}{*}{$\begin{array}{c}\text { Target } \\
\text { Self-Bias }\end{array}$} & \multicolumn{3}{|c|}{ Gas Flow } & \multirow{2}{*}{$\begin{array}{c}\text { Substrate } \\
\text { Temperature }\end{array}$} & \multirow{2}{*}{$\begin{array}{l}\text { Deposition } \\
\text { Rate }\end{array}$} & \multirow{2}{*}{ Thickness } & \multirow{2}{*}{$\begin{array}{l}\text { Optical } \\
\text { Gap }\end{array}$} \\
\hline & & & & $\mathbf{A r}$ & $\mathbf{H}_{2}$ & $\mathbf{N}_{2}$ & & & & \\
\hline & & (W) & $(\mathrm{V})$ & & $(\mathrm{sccm})$ & & $(\mathrm{K})$ & $(\AA / \mathrm{\AA} / \mathrm{s})$ & $(\mathrm{nm})$ & $(\mathrm{eV})$ \\
\hline sp21 & GaN:H & 45 & 96 & 10.0 & 5.0 & 15.0 & 330 & 1.21 & 1523 & 3.67 \\
\hline sp22 & GaN:H & 60 & 104 & 7.5 & 2.5 & 20.0 & 330 & 1.54 & 1656 & 3.60 \\
\hline sp23 & GaN & 75 & 145 & 0.0 & 0.0 & 20.0 & 330 & 1.82 & 1921 & 3.21 \\
\hline sp24 & GaN:H & 75 & 120 & 0.0 & 5.0 & 20.0 & 330 & 1.91 & 2069 & 3.72 \\
\hline sp30 & GaN & 30 & 92 & 10.0 & 0.0 & 20.0 & 420 & 0.43 & 808 & 3.26 \\
\hline sp31 & GaN:H & 30 & 76 & 7.5 & 2.5 & 20.0 & 420 & 0.31 & 361 & 3.51 \\
\hline sp32 & GaN:H & 60 & 128 & 7.5 & 15.0 & 20.0 & 330 & 1.19 & 439 & 4.11 \\
\hline sp33 & GaN & 60 & 140 & 7.5 & 0.0 & 20.0 & 330 & 0.92 & 335 & 3.07 \\
\hline sp34 & GaN:H & 60 & 125 & 7.5 & 0.3 & 20.0 & 330 & 1.91 & 689 & 3.71 \\
\hline
\end{tabular}

silica- $\mathrm{SiO}_{2}$ ) were used simultaneously. The substrate temperature was measured by a thermocouple located at the deposition face of a fused $\mathrm{SiO}_{2}$ substrate and it was $420 \mathrm{~K}$ for the samples sp30 and sp31, and was $330 \mathrm{~K}$ for all others samples. Table I shows the other deposition parameters and the optical gap of each sample.

The structural characterization was performed by X-ray diffraction at grazing $\left(3^{\circ}\right)$ incidence and $\theta-2 \theta$ detection geometry. The diffractometer (Rigaku - Ultima+ 2000) uses a CuK $\alpha 1$ radiation $(1.54056 \AA)$. The Scherrer's formula [12] was used to roughly estimate the mean crystallite sizes, without any further refinement. The atomic force microscope (AFM) measurements were performed in air. A Park Scientific Instruments AutoProbe $C P$ setup was used in contact mode with a $20 \mathrm{~nm}$ diameter silicon tip.

The optical normal incidence transmittance and near normal ( $8^{\circ}$ off-normal) reflectance spectra were measured in the $190-900 \mathrm{~nm}$ range in a Varian Cary-1 spectrophotometer equipped with integrating sphere apparatus. The spectrophotometric data were used to calculate the refractive index, absorption coefficient and film thickness, according to computer routines based on existing methods $[13,14]$.

\section{RESULTS}

The obtained films presented good adherence to the substrate and good optical homogeneity. The surface roughness was analyzed by AFM (Fig. 2). By comparison with results reported for other materials [15], the observed surface roughness was associated to a quenched growth regime [15]. In this regime the adsorbing species do not have sufficient mobility on the surface during film growth to soften the surface. The resulting statistical roughness is probably due to the relatively low ratio of substrate temperature to the GaN melting temperature $(\mathrm{Ts} /$ Tmelt $\sim 0.17)$ and also to the low kinetic energy of the adsorbing species. The low kinetic energy is due to the moderate RF power/accelerating bias potential used (Table I) and to the thermallization of the energetic $\mathrm{Ga}$ atoms ejected from the target by with collisions with the working gas mix- ture.

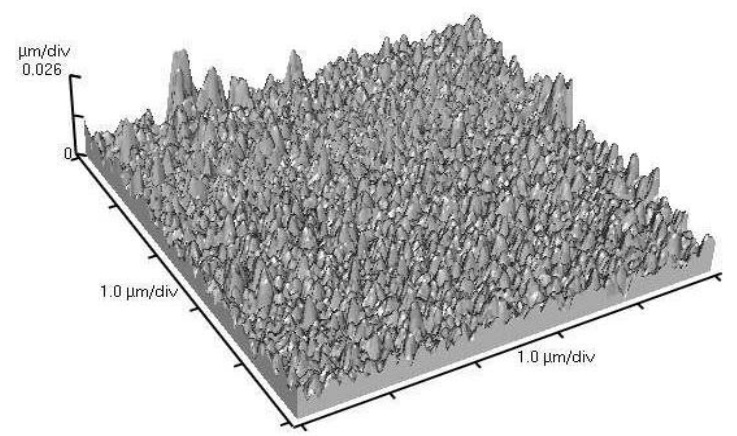

FIG. 2: AFM image of a GaN:H/Si(100) sample (sp34). Observe that the base plane scales are much wider than the vertical scale.

Concerning the atomic arrangements of the film material, the X-ray diffraction patterns, Fig.3, indicates that the non-hydrogenated $\mathrm{GaN}$ film grown on amorphous $\mathrm{SiO}_{2}$ have a considerable crystallized volume fraction. The observed diffraction peaks indicate that the crystallites of the nonhydrogenated material have mean grain sizes of the order of $15 \mathrm{~nm}$, wurtzite structure $(\alpha-\mathrm{GaN})$, and a tendency of preferential growth of the (0002) planes. Very similar results were observed for our films grown on $\mathrm{Si}(100)$ substrates. In this case the only difference is that the films grown on Si generally presented slightly stronger $(\sim 15-20 \%)$ peaks, indicating a weak tendency to be more crystallized than the ones grown in amorphous silica $\left(\mathrm{SiO}_{2}\right)$ substrates.

Analyzing samples with similar deposition parameters except for the hydrogen flux, it was noticed that the presence of hydrogen in the plasma reduces the grain size and the crystallization fraction of the films grown on $\mathrm{SiO}_{2}$ substrates (Fig.3). The same behavior was observed on hydrogenated films grown on Si substrates (not shown). When comparing X-ray diffraction results obtained for samples sp23 (nonhydrogenated) and sp24 (hydrogenated) with those of samples sp30 (non-hydrogenated) and sp31 (hydrogenated), prepared under lower RF power and higher substrate tempera- 
ture $(420 \mathrm{~K})$, and with samples sp33 (non-hydrogenated) and sp32/sp34 (hydrogenated), prepared under lower RF and different $\mathrm{H}_{2}$ gas dilutions, a similar trend was observed: the nonhydrogenated films exhibit diffractograms compatible with a significant amount of crystallization, while the diffractograms of hydrogenated samples are almost completely broad indicating amorphous characteristic. It is important to emphasize that this was valid even for the smaller $\mathrm{H}_{2}$ dilution fraction allowed by our setup (sp34). So, concerning the resulting structural characteristics of the samples, the presence of hydrogen had the higher influence among all the deposition parameters.

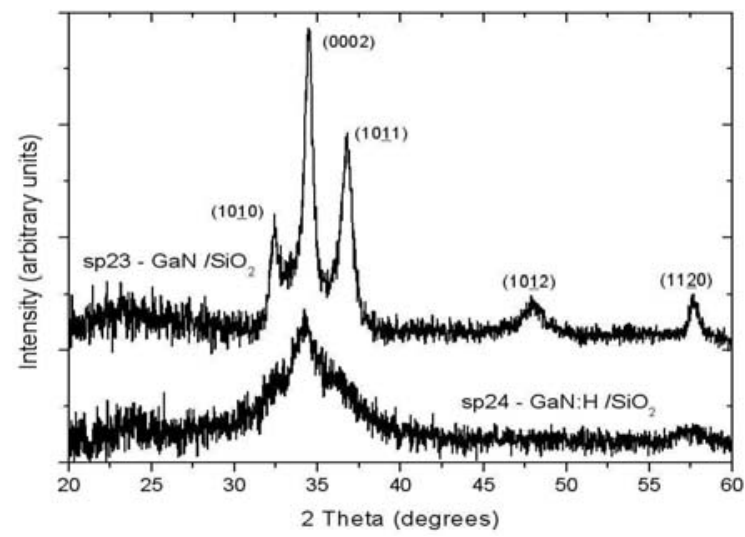

FIG. 3: X-ray diffraction patterns of the sp23 (non-hydrogenated) and sp24 (hydrogenated) samples grown onto amorphous silica substrates.

The transmittance and reflectance spectra of the films displayed a very regular set of interference fringes in the transparent and weakly absorbing spectral regions indicating good optical homogeneity of the films, and allowing good precision $(\sim 5 \%)$ in the determination of optical constants. The calculated absorption coefficient curves in the absorption edge spectral region are displayed in Fig. 4. It is apparent from this figure that the set of non-hydrogenated samples can be distinguished of the set of hydrogenated curves, the curves in each group being similar to each other. This evidences that the effect of the presence of hydrogen on the optical absorption overcomes the effects of the variation of other deposition parameters, similarly to what was already observed in the structural characterization. The analysis of the absorption curves indicated that the non-hydrogenated samples are more absorbing in the whole spectral region studied, except only for the low absorption part of the less hydrogenated sample (sp34). Also, with this exception, the absorption characteristics of the set of hydrogenated samples were very similar the one corresponding to GaN single crystal [4].

\section{DISCUSSION}

Considering the effect of the variations of the deposition parameters on the structural and optical properties of the samples, it was noted that the presence of hydrogen has a much

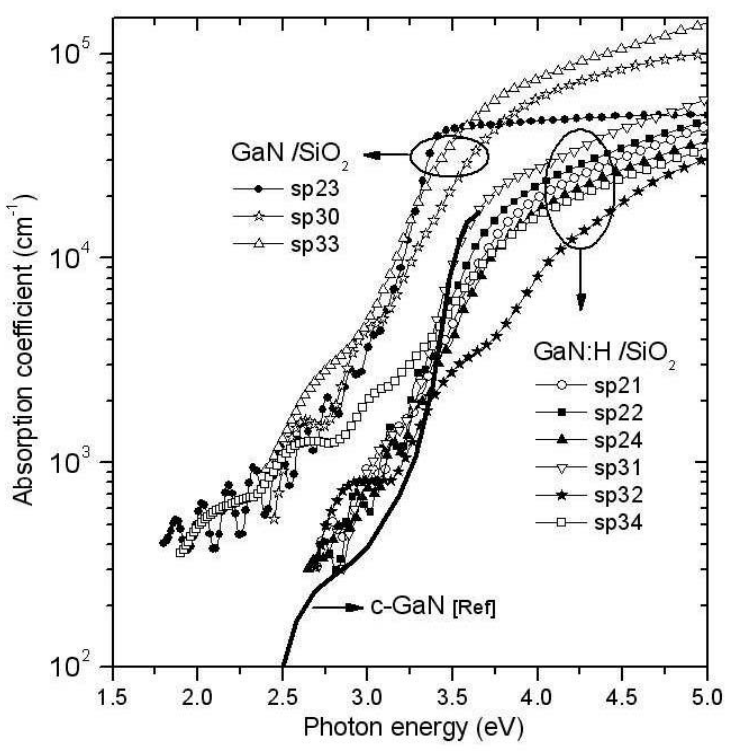

FIG. 4: Absorption edges of the GaN films. A reference edge of a single crystal GaN grown by MOCVD [4] was included for comparison.

stronger effect than other deposition parameters, namely: RF power, substrate kind (c-Si and a-SiO 2 ), substrate temperature $(330 / 420 \mathrm{~K})$, and presence of Argon in the plasma, within the ranges used in this work. The effective presence of bonded hydrogen in the films was confirmed by infrared transmittance spectra (FTIR), which display strong absorption bands associated to the $\mathrm{N}-\mathrm{H}$ stretching vibration centered at $\sim 3200 \mathrm{~cm}^{-1}$ and $\mathrm{N}-\mathrm{H}$ bending vibrations at $\sim 950 \mathrm{~cm}^{-1}$. Based on a-SiN:H oscillator strength data [16], a rough estimation of the N-H bond density was carried in the hydrogenated GaN films. This estimation indicated that the percent $\mathrm{N}-\mathrm{H}$ bond concentration can be as high as a few tens in the more hydrogenated samples. No systematic change in the growing rate or refractive index was observed in association with this relatively high hydrogen incorporation.

Comparing the optical absorption and the X-ray diffraction results, it was observed that, although the non-hydrogenated films had higher crystallized fractions, the absorption edge indicated that these films have higher density of gap states as compared to hydrogenated ones. Trying to identify the main contribution to the sub-gap absorption of the nonhydrogenated samples, preliminary ESR characterization was carried on both hydrogenated and non-hydrogenated films at room temperature and at $2 \mathrm{~K}$. These measurements did not detect paramagnetic centers in the samples, so the observed high density of gap states in the non-hydrogenated material were not associated to neutral dangling bonds [17], as could be expected for this significantly bond polarized material. So the associated defects should be related to charged dangling bonds or other kinds of defects. The changes in the optical absorption related to hydrogenation were probably related to the reduction of the charged defect density present in the crys- 
tallite grain boundaries or internal surfaces of voids and in the nearby amorphous regions.

Finally, it was noticed that the samples having higher crystallized fraction (the non-hydrogenated set) had also higher optical absorption above the gap energy than the amorphous set (hydrogenated samples). This fact deserves consideration since in the crystallized material the selection rules for crystalline momentum conservation would apply, and would lower the absorption for energies far from the singularity position, as compared to amorphous material. Nevertheless the small crystallite dimensions can severely interfere in the application of the symmetry selection rule due to the low dimension, and can produce severe lattice distortions and defects that are probably present. Also the creation of the defects in the crystallite grain boundaries and nearby amorphous regions would enhance the overall disorder in the material, producing the higher absorption. In this way, the lower absorption of the hydrogenated (predominantly amorphous) samples indicated a reduction of the overall disorder in the material.

Further work is necessary to a more complete understanding of the role of the deposition parameters on the films properties.

\section{CONCLUSION}

$\mathrm{GaN}$ and GaN:H films were prepared at low substrate temperatures $\left(\mathrm{T}_{S} \leq 420 \mathrm{~K}\right)$ using the reactive RF-magnetron sput- tering technique. Among the deposition parameters: different $\mathrm{N}_{2} / \mathrm{H}_{2} / \mathrm{Ar}$ gas mixtures, RF power, substrate kind ( $\mathrm{Si}$ (100), $\left.\mathrm{a}-\mathrm{SiO}_{2}\right)$, and substrate temperatures $(330 / 420 \mathrm{~K})$, the stronger effect observed on structural and optical properties was related to the presence of hydrogen in the plasma gas mixture. The presence of hydrogen in the plasma, even in small quantities, significantly reduces the crystallized fraction and crystallite sizes (in the nanometer scale range). Hydrogenation is also associated to an increase of the optical gap and a reduction of optical absorption at lower energies. The observed effects are indirect indications that the presence of hydrogen is probably related to an overall defect density reduction in crystallite grain boundaries and internal surfaces of voids in the GaN films produced by reactive sputtering.

\section{Acknowledgements}

We acknowledge Prof. Mônica Alonso Cotta and João Hermes Clerice (SPM/LPD laboratory, Unicamp) for the AFM measurements and Prof. Margarida Juri Saeki and Prof. Lígia de Oliveira Ruggiero for the use of the FTIR and UV-Vis spectrophotometers respectively. This work was supported by FAPESP.
[1] S. J. Pearton (ed.). GaN and Related Materials. Gordon Breach Science Publishers, Amsterdam, 1997, 538p.

[2] S. C. Jain, M. Willander, J. Narayan, R. Van Overstraeten. Journal of Applied Physics 87, 965 (2000).

[3] S. J. Pearton, C. R. Abernathy, M. E. Overberg, et.al. Journal of Applied Physics 93(1), 1 (2003).

[4] S. Strite and H. Morkoç. Journal of Vacuum Society and Technology B 10(4), 1237, (1992).

[5] J. Kim and P. H. Holloway. Journal of Vacuum Society and Technology A 22(4), 1591 (2004).

[6] T. Miyazaki, K. Takada, S Adachi, and K. Ohtsuka. Journal of Applied Physics 97, 093516 (2005).

[7] S. B. Aldabergenova, A. Osvet, G. Frank, H. P. Strunk, P. C. Taylor, and A. A. Adreev, Journal of Non-Crystalline. Solids 299-302, 709 (2002).

[8] Q. X. Guo, A. Okada, H. Kidera, T. Tanaka, M. Nishio, and H. Ogawa, Journal of Crystal Growth 237, 1079 (2002).

[9] S. Nakamura, N. Isawa, M. Senoh, and T. Mukai. Jpn. Journal of Applied Physics 31, 1258 (1992).

[10] S. J. Pearton. The role of hydrogen in GaN and related com- pounds. In: GaN and Related Materials. S.J. Pearton (ed.). Gordon Breach Science Publishers, Amsterdam, 1997. Chap. 11, pp. 333-369.

[11] A. L. J. Pereira, J. V. Lopez, and J. H. Dias da Silva, Revista Brasileira de Aplicações de Vácuo 22(2), 50 (2003).

[12] B. D. Cullity, S. R. Stock. Elements of X-Ray Diffraction, 3rd edition, Prentice-Hall, Inc., New Jersey, 2001, 664p.

[13] J. I. Cisneros. Applied Optics 37, 5262 (1998).

[14] J. I. Cisneros, G. B. Rego, M. Tomyiama, et al. Thin Solid Films 100(2), 155 (1983).

[15] J. A. Thornton. Journal of Vacuum Society and Technology 11 (4), 666 (1974)

[16] A. Morimoto, I. Kobayashi, M. Kumeda, and T. Shimizo. Japanese Journal of Applied Physics 25 (9), L752 (1986).

[17] The ESR characterization of GaN and GaN:H samples were made at the Grupo de Propriedades Ópticas e Magneticas do IFGW-Unicamp, in colaboration with Prof. Pascoal Pagliuso and Wellington Iwamoto. 